\title{
Re-defining the extent of malaria transmission in South Africa: Implications for chemoprophylaxis
}

\author{
N Morris, ${ }^{1}$ MSc; J Frean, ${ }^{2,3}$ MMed (Path Microbiol); L Baker, ${ }^{4}$ Dip Pharm; I S Ukpe, ${ }^{5}$ MMed (Fam Med); K I Barnes, ${ }^{6}$ MMed (Clin Pharm); \\ P Kruger, ${ }^{7}$ ND Pub Hlth; A Mabuza, ${ }^{8}$ ND Pub Hlth; E Raswiswi, ${ }^{9}$ BTech Env Hlth; R Maharaj, ${ }^{1}$ PhD; L Blumberg, ${ }^{2,3}$ MMed (Path Microbiol); \\ D Moonasar, ${ }^{10} \mathrm{DrPH}$
}

\author{
${ }^{1}$ Malaria Research Unit, South African Medical Research Council, Durban, South Africa \\ ${ }^{2}$ National Institute for Communicable Diseases, Johannesburg, South Africa \\ ${ }^{3}$ School of Pathology, University of the Witwatersrand, Johannesburg, South Africa \\ ${ }^{4}$ Amayeza Drug Information Centre, Johannesburg, South Africa \\ ${ }^{5}$ Department of Family Medicine, University of Pretoria, South Africa \\ ${ }^{6}$ Division of Clinical Pharmacology, Department of Medicine, University of Cape Town, South Africa \\ ${ }^{7}$ Department of Health, Polokwane, Limpopo, South Africa \\ ${ }^{8}$ Department of Health, Nelspruit, Mpumalanga, South Africa \\ ${ }^{9}$ Department of Health, Jozini, KwaZulu-Natal, South Africa \\ ${ }^{10}$ National Department of Health, Pretoria, South Africa
}

Corresponding author: N Morris (nmorrism@gmail.com)

\begin{abstract}
Background. Malaria case numbers reported in South Africa have reduced considerably over the last decade, necessitating a revision of the national risk map to guide malaria prevention, including the use of chemoprophylaxis.

Objectives. To update the national malaria risk map based on recent case data and to consider the implications of the new transmission profile for guiding prophylaxis.

Methods. The geographical distribution of confirmed malaria cases detected both passively and actively over the last six malaria seasons was used to redefine the geographical distribution and intensity of malaria transmission in the country.

Results. The national risk map was revised to reflect zones of transmission reduced both in their extent and their intensity. Most notably, the area of risk has been reduced in the north-western parts of Limpopo Province and is limited to the extreme northern reaches of KwaZuluNatal Province. Areas previously considered to be of high risk are now regarded to be of moderate risk.

Conclusion. Chemoprophylaxis is now only recommended from September to May in the north-eastern areas of Limpopo and Mpumalanga Provinces. The recommended options for chemoprophylaxis have not changed from mefloquine, doxycycline or atovaquone-proguanil.

S Afr Med J 2013;103(11):861-864. DOI:10.7196/SAMJ.7533
\end{abstract}

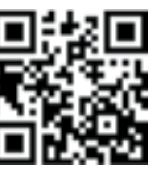

Malaria in South Africa (SA) is endemic in the northeastern reaches of the three provinces of KwaZuluNatal (KZN), Limpopo and Mpumalanga. The risk of infection peaks during the summer rainfall season, typically between September and May. The predominant malaria species is Plasmodium falciparum, transmitted by two main vector species, namely Anopheles arabiensis and Anopheles gambiae.

Malaria case notifications of $<10000$ annually between 1950 and 1990 escalated sharply during the epidemic of the 1999/2000 season, reaching over 60000 cases nationally with some 41400 of these reported in KZN. Strengthened and sustained vector control and case-management strategies using artemisinin-based combination therapy (ACT) were coupled with the implementation of a regional cross-border collaborative programme between SA, Swaziland and Mozambique aimed at extending effective vector control and malaria diagnosis and treatment into border areas of neighbouring countries. ${ }^{[1]}$ Massive reductions were realised in all sectors, most notably by some 90\% in northern KZN between 1999 and 2004. ${ }^{[2,3]}$

SA has actively controlled malaria since the 1940s using the World Health Organization (WHO) recommended strategies of: vector control; case management; surveillance; and information, education and communication (IEC) ${ }^{[4]}$ Through these interventions, SA has succeeded in reducing the burden of the disease and is now targeting malaria elimination (zero local malaria cases) by the year $2018 .^{[5]}$
It is imperative that healthcare workers are adequately informed of appropriate drug and non-drug measures for the prevention of malaria. In keeping with the National Guidelines for Malaria Prevention, healthcare workers need to know where travellers intend to visit, before recommending appropriate preventive strategies and prescribing chemoprophylaxis, if indicated. ${ }^{[6]}$ An up-to-date malaria risk map is therefore required to guide chemoprophylactic and nondrug protective measures.

The national malaria risk map, last updated in 2007, has been revised based on recent data and reflects risk zones that are reduced both in geographical extent and malaria transmision intensity. Redefining the malaria risk profile in SA provides an opportunity to revisit national recommendations for precaution and prevention.

\section{Objective}

We define the criteria used to review the malaria risk map for SA and provide advice on chemoprophylactic and non-drug measures for the prevention of the disease.

\section{Methods}

Malaria risk in SA was depicted in 1938 as affecting much of (then) Natal and the northern and eastern lowveld, extending as far as Port St Johns in the south and Pretoria and the Orange River in the north-west 


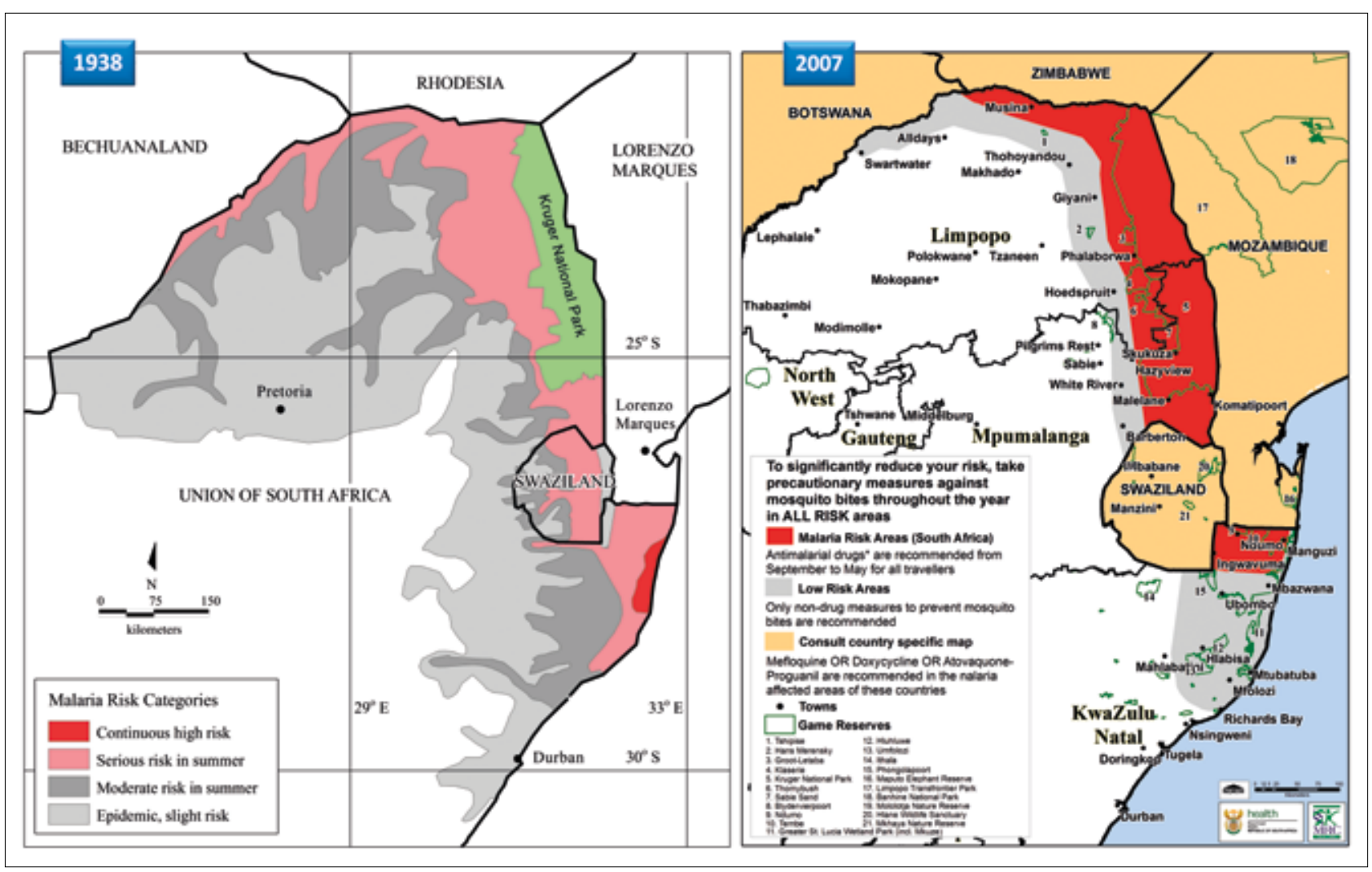

Fig. 1. Malaria risk in 1938 prior to the implementation of control measures (left) and malaria risk in 2007 following the implementation of regional crossborder control (right).

(Fig. 1). ${ }^{[7]}$ The official map depicting malaria risk in the country was next revised in 2000, informed by case notifications and evidence of the presence of suitable vectors, and depicted malaria confined largely to the north-east of the country, bound in the south by Richards Bay and in the north-east by Louis Trichardt and Swartwater.

Minor adjustment to the malaria risk map was made in 2007. This involved drawing the risk profile as starting further north in KZN where it was limited to the three northern districts, namely uMkhanyakude, Uthungulu and Zululand. Areas as far north as Lake St Lucia were declared malaria-free (Fig. 1) ${ }^{[8]}$ At this stage, notified malaria case aggregates at sub-district level provided the primary indicator of the geographical extent of reported local infections.

The national malaria case burden has continued to see sustained reductions to around 10000 annually for the last five years following a systematic programme of strengthening vector control coupled with case management using ACT, and a reduction of both the parasite and vector reservoirs in neighbouring Swaziland and Mozambique. All nine malarious districts of SA met elimination criteria in 2013, reporting $<1$ case per 1000 population at risk.

Critical to the success of the control programme, and indeed the elimination agenda going forward, has been the implementation of a spatially-enabled malaria information system (MIS). Individual patient case data passively notified via health facilities and infections actively detected by surveillance agents in communities are recorded within the MIS. ${ }^{[9]}$ Malaria cases reported in the MIS have historically been mapped at district level, with the exception of KZN where small-scale malaria planning area boundaries were developed in 1999 using a combination of global positioning system (GPS) coordinate data and topographical reference mapping.

A large-scale initiative to map malaria case data reported through the provincial MISs at the level of residential locality of all cases notified since 1996, was embarked upon in 2004 and completed in 2011. This enabled high-resolution mapping of malaria nationally for the first time in 2012. This initiative provided a unique opportunity to update the national malaria risk map, consulting notified case data at high spatial resolution. Cases were mapped at the locality level for the last thirteen malaria seasons and stratified by their probable source of infection. Predominant clusters of locally-acquired infections over the last five seasons were used to determine zones of malaria risk in the country. Two broad categories of low- and moderate-risk areas were defined in keeping with the dramatically reduced case burden in the country since the drawing of the 2007 version of the risk map.

The revised malaria risk profile boundary was developed, based on malaria incidence rates over the past five years at municipality level, and then adjusted intra-municipally, taking cognisance of known malaria incidence at locality level.

\section{Results}

In June 2013, the South African Malaria Elimination Committee (SAMEC) reached agreement over the geographical distribution and intensity of malaria risk captured in the updated malaria risk map (Fig. 2).

The key changes from the 2007 version of the risk map include the following:

1. The northern-most area of KZN previously denoted as a 'risk area' is now classified as a 'low risk area'. The low risk area previously extending as far south as Richards Bay has now been scaled back to end further north around the Mtubatuba and Mfolozi areas.

2. The low risk profile in the northern provinces of Limpopo and Mpumalanga has been scaled back in line with a reduced incidence of local malaria case notifications observed in the western parts of these provinces. Most notably, the low risk area in Limpopo, 


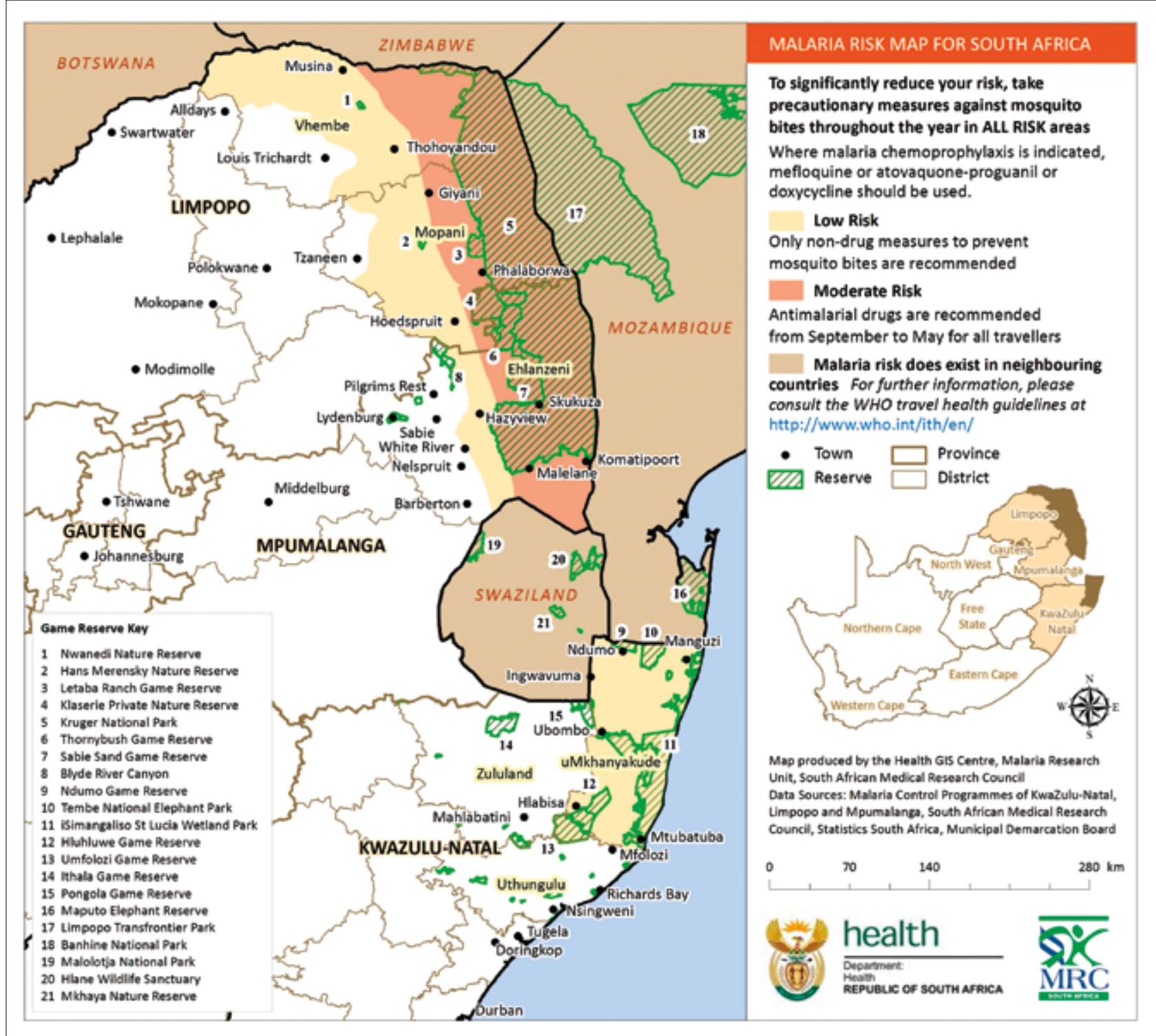

Fig. 2. Official malaria risk map for South Africa, 2013.

previously extending as far west as Swartwater, now ends east of Alldays, with the moderate risk area ending east of Musina. Although a fair number of cases are reported at Musina, they are typically imported infections associated with border crossings in the proximity of the town.

3. Previous guidance appearing on the risk map referring visitors to country-specific maps for further risk profile information for neighbouring countries has been amended to refer instead to the WHO international travel health guidelines.

4. The colour scheme and terminology of the risk classes have been adjusted in line with the substantial reductions in malaria risk observed nationally. The high risk area previously in red has been scaled back as noted in (1) and (2) above, and is now referred to as a 'moderate risk' area depicted in a lighter shade.

5. An inset map of SA has been added on the right indicating the malarious provinces and risk areas within national bounds.

The official risk map, now endorsed by the National Department of Health, has been updated with due consideration given to actual notified case data in the malarious provinces, and takes into account the intended audience, namely those providing travel advice.

\section{Discussion \\ Implications for prevention}

The decision of whether to recommend malaria chemoprophylaxis to a traveller requires consideration of the risk of contracting malaria in the specific area at the specific time, as well as the risk of adverse effects of any chemoprophylaxis prescribed. The revised malaria risk map, based on actual notified case data, enables the health professional to assess more accurately the risk to the traveller of contracting malaria. Previously, chemoprophylaxis was recommended during the warm wet season in the northern parts of KZN and in the north-eastern areas of Limpopo and Mpumalanga. Based on the new data, chemoprophylaxis is now only recommended from September to May in the north-eastern areas of Limpopo and Mpumalanga.

The recommended options for chemoprophylaxis have not changed and remain the following: ${ }^{[6]}$ 
- Mefloquine - from $5 \mathrm{~kg}$ (or 3 months of age), a weekly dose started at least 1 week prior to entering the malaria area, once weekly while there and once weekly for 4 weeks after leaving the area.

- Doxycycline - from 8 years of age, a daily dose started at least 1 day before entering the area, once daily while in the area and once daily for 4 weeks after leaving the area.

- Atovaquone-proguanil - from $11 \mathrm{~kg}$, a daily dose started at least 1 day before entering the area, once daily while in the area and once daily for 7 days after leaving the area.

Stringent non-drug measures should be taken to avoid mosquito bites throughout the year, in areas of both low and moderate malariatransmission intensity. Measures that reduce contact with mosquitoes have the advantage that they are less toxic than chemoprophylactic drugs and that their effectiveness does not depend on the drug sensitivity of the parasite. These measures include: ${ }^{[6]}$

- Remaining indoors between dusk and dawn.

- Wearing long-sleeved clothing, long trousers and socks when going out at night.

- Covering doorways and windows with screens but, if not available, windows and doors should be closed at night.

- Applying an N,N-diethyl-meta-toluamide (DEET)-containing insect repellent to exposed skin; repeat as recommended on the container label. Avoid eyelids, lips, sunburnt or damaged skin, do not spray on the face and do not overdose young children.

- Using mosquito mats impregnated with an insecticide (heated electrically or by a non-electric lamp), or burning mosquito coils in living and sleeping areas during the night.

- Using a mosquito-proof bed net over the bed, with edges tucked in, ensuring that the net is not torn and that there are no mosquitoes inside. Protection will be increased by treating the net periodically with an insecticide registered for this purpose; e.g. a pyrethroid.

- Spraying inside the house with an aerosol insecticide (for flying insects) at dusk, especially the bedrooms, after closing the windows.

- Using ceiling fans and air conditioners.

- Treating clothes with an insecticide registered for this purpose; e.g. a pyrethroid.

\section{Conclusion}

An accurate national malaria risk profile is necessary to inform prophylaxis guidelines. High-resolution mapping of individual case notification data at the locality level has enabled an accurate assessment of the geographical extent of the occurrence of local infections. Substantial gains in reducing the extent and intensity of malaria transmission in SA over the last decade are now reflected in the revised national risk map. This data-driven mapping of the malaria risk profile enables rational recommendations regarding the use of non-drug and chemoprophylactic measures, thereby supporting the national malaria prevention guidelines. As malaria control interventions continue to be strengthened, and malaria elimination strategies start to be implemented, it is essential that the risk map is regularly updated. This depends on all malaria cases being notified promptly.

Acknowledgements. The authors are grateful to the provincial information officers and GIS technicians involved in producing the quality data that informed the update of the national risk map. We record our appreciation to $\mathrm{Mr}$ Ishen Seocharan for maintenance, management and integration of the MISs at the national level, without which this exercise would not have been possible. Credit is also due to the wider membership of the SAMEC, whose expert opinion has provided the backdrop to the exercise. The authors wish to acknowledge the important role of healthcare personnel and surveillance agents in case detection and data collection.

Author contributions. NM, DM, LBK, ISU, KB, RM and JF drafted the manuscript. LBL and JF reviewed and edited the manuscript. NM developed the risk map. PK, AM and ER guided fine-scale adjustments to the risk map. All authors read, contributed to, and approved the final versions of both the map and the manuscript.

\section{References}

1. Sharp BL, Kleinschmidt I, Streat E, et al. Seven years of regional malaria control collaboration Mozambique, South Africa and Swaziland. Am J Trop Med Hyg 2007;76(1):42-47.

2. Barnes KI, Durrheim DN, Little F, et al. Effect of artemether-lumefantrine policy and improved vector control on malaria burden in KwaZulu-Natal, South Africa. PLoS Medicine 2005;2(11):e330. [http:// dx.doi.org/10.1371/journal.pmed.0020330]

3. Maharaj R, Mthembu DJ, Sharp BL. Impact of DDT re-introduction on malaria transmission in KwaZulu-Natal. S Afr Med J 2005;95(11):871-874.

Sharp BL, le Sueur D. Malaria in South Africa - the past, the present and selected implications for the future. S Afr Med J 1996;86(1):83-89.

5. National Department of Health. South African National Malaria Elimination Strategic Plan 2012-2018. Pretoria: NDoH, 2012.

6. National Department of Health. Guidelines for the Prevention of Malaria in South Africa. Pretoria NDoH, 2009.

Le Sueur D, Sharp BL, Appleton CC. Historical perspective of the malaria problem in Natal with Le Sueur D, Sharp BL, Appleton CC. Historical perspective of
emphasis on the period 1928-1932. S Afr J Sci 1993;89:232-239.

emphasis on the period 1928-1932. S Afr J Sci 1993;89:232-239. . National Department

Pretoria: NDoH, 2010 .
Martin CL, Curtis B, Fraser C, et al. The use of a GIS-based malaria information system for malaria research and control in South Africa. Health Place 2002;8(4):227-236. [http://dx.doi.org/10.1016 S1353-8292(02)00008-4]

Accepted 1 October 2013 